\title{
Transverse Wave Propagation in Relativistic Two-fluid Plasmas in de Sitter Space
}

\author{
M. Atiqur Rahman 11 and M. Hossain Ali 2 \\ Department of Applied Mathematics, University of Rajshahi, Rajshahi-6205, Bangladesh.
}

\begin{abstract}
We investigate transverse electromagnetic waves propagating in a plasma in the de Sitter space. Using the $3+1$ formalism we derive the relativistic two-fluid equations to take account of the effects due to the horizon and describe the set of simultaneous linear equations for the perturbations. We use a local approximation to investigate the one-dimensional radial propagation of Alfvén and high frequency electromagnetic waves and solve the dispersion relation for these waves numerically.
\end{abstract}

Keywords: Two-fluid plasma, Alfvén and high frequency electromagnetic waves, Cosmological event horizon.

\footnotetext{
${ }^{1}$ E-mail: atirubd@yahoo.com

${ }^{2}$ The Abdus Salam International Centre for Theoretical Physics, Strada Costiera 11, 34014 Trieste, Italy. E-mail: mali@ictp.it,m_hossain_ali_bd@yahoo.com (Corresponding author).
} 


\section{Introduction}

In recent years there have been renewed interests in investigating plasmas in curved spacetimes of general relativity; because, a successful study of the waves and emissions from plasmas falling into a compact body (e.g. black hole) will be of great value in aiding the observational identification of black hole candidates. The de Sitter (dS) space with positive cosmological constant has properties similar to a black hole. We study two-fluid plasmas near the horizon of the pure dS space.

Over the last few decades, physicists have a growing interest in dS space. In the 1970s, the attention was due to the large symmetry group of dS space, which made the field theory in dS space less ambiguous than, for example, in the Schwarzschild spacetime. Researches in the 1980s focused the role it played during inflation-accelerated expansion in the very early universe. The universe is currently asymptotic dS and approach a pure dS space. Recent cosmological observations [1, 2, 3, 4, 5, 6] suggest the possibility of existing a positive cosmological constant $(\Lambda>0)$ in our universe and this possibility gives the picture, among many others, of some features closely related to black holes: the existence of cosmological event horizons. These causal horizons exist even in the absence of matter, namely in empty dS space, and hide all the events which are not accessible for geodesic observers. In addition, the success of the ADS/CFT correspondence [7, 8, 9, 10, has led to the intense study of dS space in the context of the quantum gravity [1]. The attention has been to obtain an analogue of the ADS/CFT correspondence in dS space, i.e. dS/CFT correspondence [12, 13, 14, 15, 16, 17, 18, 19, 20, 21, 22, 23, 24, 25, 26] in the light of which there has been an extensive study of the semiclassical aspects of dS and asymptotic dS spacetimes [27, 28, 29, 30, 31, 32, 33. In view of these reasons, it may be of special interest to investigate electromagnetic waves in a plasma in the dS space.

Recently, Buzzi, et.al. [33, 34], using the $3+1$ formulation [35, 36, 37, 38], described a general relativistic version of two-fluid formulation of plasma physics and investigated the nature of plasma waves (transverse waves in [33, and longitudinal waves in 34]) near the horizon of the Schwarzschild black hole. In this paper we apply the formalism of Buzzi et.al. 33. to investigate the transverse electromagnetic waves propagating in a plasma close to the (cosmological) event horizon of the pure dS space.

This paper is organized as follows. In section 2 we summarize the $3+1$ formulation of general relativity. In section 3 we describe the horizon plasma governing equations. In section 4 we consider one-dimensional wave propagation in the radial $z$ (Rindler coordinate system) direction. We linearize the equations in section 5 by considering a small perturbation to fields and fluid parameters. In section 6 we discuss the local or mean-field approximation for the lapse function $\alpha$ and obtain a dispersion relation for the transverse wave. In section 7 we describe a procedure for solving the dispersion relation numerically. In sections 8 we present our results. Finally, in section 9 we give our remarks. We use units $G=c=k_{B}=1$. 


\section{$2 \quad 3+1$ Spacetime Formalism}

In de sitter space, the simplest solution for the Einstein field equations with $T_{\mu \nu}=0$ is written as

$$
\begin{aligned}
d s^{2} & =g_{\mu \nu} d x^{\mu} d x^{\nu} \\
& =-\left(1-\frac{r^{2}}{\ell^{2}}\right) d t^{2}+\left(1-\frac{r^{2}}{\ell^{2}}\right)^{-1} d r^{2}+r^{2} d \Omega_{2}^{2} .
\end{aligned}
$$

Here, $\ell$ is the curvature radius of the dS space [i.e., $\Lambda=\frac{3}{\ell^{2}}$ is the positive cosmological constant], $d \Omega_{2}^{2}$ represents a unit 2 -sphere, and the nonangular coordinates range according to $0 \leq r \leq \ell$ and $-\infty \leq t \leq \infty$. The boundary at $r=\ell$ describes a cosmological horizon for an observer located at $r=0$.

An absolute three-dimensional space defined by the hypersurfaces of constant universal time $t$ is described by the metric

$$
d s^{2}=g_{i j} d x^{i} d x^{j}=\left(1-\frac{r^{2}}{\ell^{2}}\right)^{-1} d r^{2}+r^{2} d \Omega_{2}^{2} .
$$

The indices $i, j$ range over $1,2,3$ and refer to coordinates in absolute space. The Fiducial Observers (FIDOs), the observers remaining at rest with respect to this absolute space, measure their proper time $\tau$ using clocks that they carry with them and make local measurements of physical quantities. Then all their measured quantities are defined as FIDO locally measured quantities and all rates measured by them are measured using FIDO proper time. The FIDOs use a local Cartesian coordinate system with unit basis vectors tangent to the coordinate line

$$
\mathbf{e}_{\hat{r}}=\left(1-\frac{r^{2}}{\ell^{2}}\right)^{1 / 2} \frac{\partial}{\partial r}, \quad \mathbf{e}_{\hat{\theta}}=\frac{1}{r} \frac{\partial}{\partial \theta}, \quad \mathbf{e}_{\hat{\varphi}}=\frac{1}{r \sin \theta} \frac{\partial}{\partial \varphi} .
$$

For a spacetime viewpoint rather than a $3+1$ split of spacetime, the set of orthonormal vectors also includes the basis vector for the time coordinate given by

$$
\mathbf{e}_{\hat{0}}=\frac{d}{d \tau}=\frac{1}{\alpha} \frac{\partial}{\partial t}
$$

where $\alpha$ is the lapse function (or redshift factor) defined by

$$
\alpha(r) \equiv \frac{d \tau}{d t}=\left(1-\frac{r^{2}}{\ell^{2}}\right)^{1 / 2}
$$

The gravitational acceleration felt by a FIDO is given by [35, 36, 37, 38,

$$
\mathbf{a}=-\nabla \ln \alpha=\frac{1}{\alpha} \frac{r}{\ell^{2}} \mathbf{e}_{\hat{r}}
$$

while the rate of change of any scalar physical quantity or any three-dimensional vector or tensor, as measured by a FIDO, is defined by the convective derivative

$$
\frac{D}{D \tau} \equiv\left(\frac{1}{\alpha} \frac{\partial}{\partial t}+\mathbf{v} \cdot \nabla\right),
$$

$\mathbf{v}$ being the velocity of a fluid as measured locally by a FIDO. 


\section{Two-Fluid Plasma Equations in $3+1$ Formalism}

We consider a two-component plasma consisting of electrons and either positrons or ions. In the $3+1$ notation, the continuity equation for each of the fluid species is

$$
\frac{\partial}{\partial t}\left(\gamma_{s} n_{s}\right)+\nabla \cdot\left(\alpha \gamma_{s} n_{s} \mathbf{v}_{s}\right)=0
$$

where $s$ is 1 for electrons and 2 for positrons (or ions). For a perfect relativistic fluid of species $s$ in three-dimensions, the energy density $\epsilon_{s}$, the momentum density $\mathbf{S}_{s}$, and stress-energy tensor $W_{s}^{j k}$ are given by

$$
\epsilon_{s}=\gamma_{s}^{2}\left(\varepsilon_{s}+P_{s} \mathbf{v}_{s}^{2}\right), \quad \mathbf{S}_{s}=\gamma_{s}^{2}\left(\varepsilon_{s}+P_{s}\right) \mathbf{v}_{s}, \quad W_{s}^{j k}=\gamma_{s}^{2}\left(\varepsilon_{s}+P_{s}\right) v_{s}^{j} v_{s}^{k}+P_{s} g^{j k}
$$

where $\mathbf{v}_{s}$ is the fluid velocity, $n_{s}$ is the number density, $P_{s}$ is the pressure, and $\varepsilon_{s}$ is the total energy density defined by

$$
\varepsilon_{s}=m_{s} n_{s}+P_{s} /\left(\gamma_{g}-1\right)
$$

The gas constant $\gamma_{g}$ is $4 / 3$ for $T \rightarrow \infty$ and $5 / 3$ for $T \rightarrow 0$.

Using the conservation of entropy, the equation of state can be expressed by

$$
\frac{D}{D \tau}\left(\frac{P_{s}}{n_{s}^{\gamma_{g}}}\right)=0
$$

where $D / D \tau=(1 / \alpha) \partial / \partial t+\mathbf{v}_{s} \cdot \nabla$. The full equation of state for a relativistic fluid, as measured in the fluid's rest frame, is as follows [39, 40]:

$$
\varepsilon=m_{s} n_{s}+m_{s} n_{s}\left[\frac{P_{s}}{m_{s} n_{s}}-\frac{\mathrm{i} H_{2}^{(1)^{\prime}}\left(\mathrm{i} m_{s} n_{s} / P_{s}\right)}{\mathrm{i} H_{2}^{(1)}\left(\mathrm{i} m_{s} n_{s} / P_{s}\right)}\right],
$$

where the $H_{2}^{(1)}(x)$ are Hankel functions.

The quantities of (9) in the electromagnetic field are expressed by

$$
\begin{aligned}
\epsilon_{s} & =\frac{1}{8 \pi}\left(\mathbf{E}^{2}+\mathbf{B}^{2}\right), \quad \mathbf{S}_{s}=\frac{1}{4 \pi} \mathbf{E} \times \mathbf{B}, \\
W_{s}^{j k} & =\frac{1}{8 \pi}\left(\mathbf{E}^{2}+\mathbf{B}^{2}\right) g^{j k}-\frac{1}{4 \pi}\left(E^{j} E^{k}+B^{j} B^{k}\right) .
\end{aligned}
$$

The equations for the conservation of energy and momentum are respectively given by [35, 36, 37.

$$
\begin{aligned}
\frac{1}{\alpha} \frac{\partial}{\partial t} \epsilon_{s} & =-\nabla \cdot \mathbf{S}_{s}+2 \mathbf{a} \cdot \mathbf{S}_{s}, \\
\frac{1}{\alpha} \frac{\partial}{\partial t} \mathbf{S}_{s} & =\epsilon_{s} \mathbf{a}-\frac{1}{\alpha} \nabla \cdot\left(\alpha \overleftrightarrow{\mathbf{W}}_{s}\right) .
\end{aligned}
$$

When the two-fluid plasma couples to the electromagnetic fields, the Maxwell's equations take the following $3+1$ form:

$$
\begin{aligned}
\nabla \cdot \mathbf{B} & =0, \\
\nabla \cdot \mathbf{E} & =4 \pi \sigma, \\
\frac{\partial \mathbf{B}}{\partial t} & =-\nabla \times(\alpha \mathbf{E}), \\
\frac{\partial \mathbf{E}}{\partial t} & =\nabla \times(\alpha \mathbf{B})-4 \pi \alpha \mathbf{J},
\end{aligned}
$$


where the charge and current densities are respectively defined by

$$
\sigma=\sum_{s} \gamma_{s} q_{s} n_{s}, \quad \mathbf{J}=\sum_{s} \gamma_{s} q_{s} n_{s} \mathbf{v}_{s}
$$

Using (10) and (16 19), the energy and momentum conservation equations (14) and (15) can be rewritten for each species $s$ in the form

$$
\begin{gathered}
\frac{1}{\alpha} \frac{\partial}{\partial t} P_{s}-\frac{1}{\alpha} \frac{\partial}{\partial t}\left[\gamma_{s}^{2}\left(\varepsilon_{s}+P_{s}\right)\right]-\nabla \cdot\left[\gamma_{s}^{2}\left(\varepsilon_{s}+P_{s}\right) \mathbf{v}_{s}\right] \\
+\gamma_{s} q_{s} n_{s} \mathbf{E} \cdot \mathbf{v}_{s}+2 \gamma_{s}^{2}\left(\varepsilon_{s}+P_{s}\right) \mathbf{a} \cdot \mathbf{v}_{s}=0 \\
\gamma_{s}^{2}\left(\varepsilon_{s}+P_{s}\right)\left(\frac{1}{\alpha} \frac{\partial}{\partial t}+\mathbf{v}_{s} \cdot \nabla\right) \mathbf{v}_{s}+\nabla P_{s}-\gamma_{s} q_{s} n_{s}\left(\mathbf{E}+\mathbf{v}_{s} \times \mathbf{B}\right) \\
+\mathbf{v}_{s}\left(\gamma_{s} q_{s} n_{s} \mathbf{E} \cdot \mathbf{v}_{s}+\frac{1}{\alpha} \frac{\partial}{\partial t} P_{s}\right)+\gamma_{s}^{2}\left(\varepsilon_{s}+P_{s}\right)\left[\mathbf{v}_{s}\left(\mathbf{v}_{s} \cdot \mathbf{a}\right)-\mathbf{a}\right]=0 .
\end{gathered}
$$

Although these equations are valid in a FIDO frame, they reduce for $\alpha=1$ to the corresponding special relativistic equations [41] which are valid in a frame in which both fluids are at rest. The transformation from the FIDO frame to the comoving (fluid) frame involves a boost velocity, which is simply the freefall velocity, given by

$$
v_{\mathrm{ff}}=\left(1-\alpha^{2}\right)^{\frac{1}{2}} .
$$

Then the relativistic Lorentz factor $\gamma_{\mathrm{boost}} \equiv\left(1-v_{\mathrm{ff}}^{2}\right)^{-1 / 2}=1 / \alpha$.

For a good approximation near the horizon, we write the dS metric in the Rindler coordinate system as follows:

$$
d s^{2}=-\left(1-\frac{r^{2}}{\ell^{2}}\right) d t^{2}+d x^{2}+d y^{2}+d z^{2},
$$

where

$$
x=\ell\left(\theta-\frac{\pi}{2}\right), \quad y=\ell \varphi, \quad z=2 \ell\left(1-\frac{r^{2}}{\ell^{2}}\right)^{1 / 2} .
$$

The standard lapse function in Rindler coordinates becomes $\alpha=z / 2 r_{h}$, where $r_{h}=\ell$ is the location of the cosmological event horizon.

\section{Radial Wave Propagation in One-Dimension}

We consider one-dimensional wave propagation in the radial $z$ direction and introduce the complex variables

$$
\begin{array}{r}
v_{s z}(z, t)=u_{s}(z, t), \quad v_{s}(z, t)=v_{s x}(z, t)+\mathrm{i} v_{s y}(z, t), \\
B(z, t)=B_{x}(z, t)+\mathrm{i} B_{y}(z, t), \quad E(z, t)=E_{x}(z, t)+\mathrm{i} E_{y}(z, t) .
\end{array}
$$

Then

$$
\begin{aligned}
v_{s x} B_{y}-v_{s y} B_{x} & =\frac{\mathrm{i}}{2}\left(v_{s} B^{*}-v_{s}^{*} B\right), \\
v_{s x} E_{y}-v_{s y} E_{x} & =\frac{\mathrm{i}}{2}\left(v_{s} E^{*}-v_{s}^{*} E\right),
\end{aligned}
$$


where the $*$ denotes the complex conjugate. The continuity equation (8) takes the form

$$
\frac{\partial}{\partial t}\left(\gamma_{s} n_{s}\right)+\frac{\partial}{\partial z}\left(\alpha \gamma_{s} n_{s} u_{s}\right)=0
$$

while Poisson's equation (17) becomes

$$
\frac{\partial E_{z}}{\partial z}=4 \pi\left(q_{1} n_{1} \gamma_{1}+q_{2} n_{2} \gamma_{2}\right)
$$

The $\mathbf{e}_{\hat{x}}$ and $\mathbf{e}_{\hat{y}}$ components of (18) and (19) give

$$
\begin{aligned}
\frac{1}{\alpha} \frac{\partial B}{\partial t} & =-\mathrm{i}\left(\frac{\partial}{\partial z}-a\right) E \\
\mathrm{i} \frac{\partial E}{\partial t} & =-\alpha\left(\frac{\partial}{\partial z}-a\right) B-\mathrm{i} 4 \pi e \alpha\left(\gamma_{2} n_{2} v_{2}-\gamma_{1} n_{1} v_{1}\right) .
\end{aligned}
$$

Differentiating equation (31) with respect to $t$ and using (30), we obtain

$$
\left(\alpha^{2} \frac{\partial^{2}}{\partial z^{2}}+\frac{3 \alpha}{2 r_{h}} \frac{\partial}{\partial z}-\frac{\partial^{2}}{\partial t^{2}}+\frac{1}{4 r_{h}^{2}}\right) E=4 \pi e \alpha \frac{\partial}{\partial t}\left(n_{2} \gamma_{2} v_{2}-n_{1} \gamma_{1} v_{1}\right) .
$$

The transverse component of the momentum conservation equation is obtained from the $\mathbf{e}_{\hat{x}}$ and $\mathbf{e}_{\hat{y}}$ components of (22) as follows:

$$
\rho_{s} \frac{D v_{s}}{D \tau}=q_{s} n_{s} \gamma_{s}\left(E-\mathrm{i} v_{s} B_{z}+\mathrm{i} u_{s} B\right)-u_{s} v_{s} \rho_{s} a-v_{s}\left(q_{s} n_{s} \gamma_{s} \mathbf{E} \cdot \mathbf{v}_{s}+\frac{1}{\alpha} \frac{\partial P_{s}}{\partial t}\right)
$$

where

$$
\mathbf{E} \cdot \mathbf{v}_{s}=\frac{1}{2}\left(E v_{s}^{*}+E^{*} v_{s}\right)+E_{z} u_{s}
$$

and $\rho_{s}$ is the total energy density defined by

$$
\rho_{s}=\gamma_{s}^{2}\left(\varepsilon_{s}+P_{s}\right)=\gamma_{s}^{2}\left(m_{s} n_{s}+\Gamma_{g} P_{s}\right)
$$

with $\Gamma_{g}=\gamma_{g} /\left(\gamma_{g}-1\right)$.

\section{Linearization}

We use perturbation method to linearize the equations derived in the preceding section by introducing the quantities

$$
\begin{array}{rll}
u_{s}(z, t)=u_{o s}(z)+\delta u_{s}(z, t), & v_{s}(z, t)=\delta v_{s}(z, t), \\
n_{s}(z, t)=n_{o s}(z)+\delta n_{s}(z, t), & P_{s}(z, t)=P_{o s}(z)+\delta P_{s}(z, t), \\
\rho_{s}(z, t)=\rho_{o s}(z)+\delta \rho_{s}(z, t), & \mathbf{E}(z, t)=\delta \mathbf{E}(z, t), \\
\mathbf{B}_{z}(z, t)=\mathbf{B}_{o}(z)+\delta \mathbf{B}_{z}(z, t), & \mathbf{B}(z, t)=\delta \mathbf{B}(z, t),
\end{array}
$$

where magnetic field is chosen to lie along the radial $\mathbf{e}_{\hat{z}}$ direction. The relativistic Lorentz factor is also linearized such that

$$
\gamma_{s}=\gamma_{o s}+\delta \gamma_{s}, \quad \text { where } \quad \gamma_{o s}=\left(1-\mathbf{u}_{o s}^{2}\right)^{-\frac{1}{2}}, \quad \delta \gamma_{s}=\gamma_{o s}^{3} \mathbf{u}_{o s} \cdot \delta \mathbf{u}_{s}
$$


Near the horizon the unperturbed radial velocity for each species as measured by a FIDO along $\mathbf{e}_{\tilde{z}}$ is assumed to be the freefall velocity so that

$$
u_{o s}(z)=v_{\mathrm{ff}}(z)=\left[1-\alpha^{2}(z)\right]^{\frac{1}{2}} .
$$

It follows, from the continuity equation (28), that

$$
r^{2} \alpha \gamma_{o s} n_{o s} u_{o s}=\text { const. }=r_{h}^{2} \alpha_{h} \gamma_{h} n_{h} u_{h},
$$

where the values with a subscript $h$ are the limiting values at the horizon. The freefall velocity at the horizon becomes unity so that $u_{h}=1$. Since $u_{o s}=v_{\mathrm{ff}}, \gamma_{o s}=1 / \alpha$; hence, $\alpha \gamma_{o s}=\alpha_{h} \gamma_{h}=1$. Also, because $v_{\mathrm{ff}}=r / r_{h}$, the number density for each species can be written as follows:

$$
n_{o s}(z)=n_{h s} v_{\mathrm{ff}}^{-3} .
$$

The equation of state (11) and (38) lead to write the unperturbed pressure, in terms of the freefall velocity, as follows:

$$
P_{o s}(z)=P_{h s} v_{\mathrm{ff}}^{-3 \gamma_{g}} .
$$

Since $P_{o s}=k_{B} n_{o s} T_{o s}$, then with $k_{B}=1$, the temperature profile is

$$
T_{o s}=T_{h s} v_{\mathrm{ff}}^{-3\left(\gamma_{g}-1\right)}(z) .
$$

The unperturbed magnetic field is purely in the radial direction and it does not experience effects of spatial curvature. From the flux conservation $\nabla \cdot \mathbf{B}_{o}=0$ it follows that

$$
r^{2} B_{o}(r)=\text { const. }
$$

One can obtain from this the unperturbed magnetic field, in terms of the freefall velocity, as follows:

$$
B_{o}(z)=B_{h} v_{\mathrm{ff}}^{-2},
$$

where $v_{\mathrm{ff}}=\left[1-\alpha^{2}(z)\right]^{1 / 2}$. Since

$$
\frac{d v_{\mathrm{ff}}}{d z}=-\frac{\alpha}{2 r_{h}} \frac{1}{v_{\mathrm{ff}}},
$$

we have

$$
\begin{array}{ll}
\frac{d u_{o s}}{d z}=-\frac{\alpha}{2 r_{h}} \frac{1}{v_{\mathrm{ff}}}, & \frac{d B_{o}}{d z}=\frac{\alpha}{r_{h}} \frac{B_{o}}{v_{\mathrm{ff}}^{2}}, \\
\frac{d n_{o s}}{d z}=\frac{3 \alpha}{2 r_{h}} n_{o s}, & \frac{d P_{o s}}{d z}=\frac{3 \alpha}{2 r_{h}} \frac{\gamma_{g} P_{o s}}{v_{\mathrm{ff}}^{2}} .
\end{array}
$$

When the linearized variables from (35) and (36) are substituted into the continuity equation and products of perturbation terms are neglected, the result gives

$$
\begin{aligned}
& \gamma_{o s}\left(\frac{\partial}{\partial t}+u_{o s} \alpha \frac{\partial}{\partial z}+\frac{u_{o s}}{2 r_{h}}+\gamma_{o s}^{2} \alpha \frac{d u_{o s}}{d z}\right) \delta n_{s}+\left(\alpha \frac{\partial}{\partial z}+\frac{1}{2 r_{h}}\right)\left(n_{o s} \gamma_{o s} u_{o s}\right) \\
& +n_{o s} \gamma_{o s}^{3}\left[u_{o s} \frac{\partial}{\partial t}+\alpha \frac{\partial}{\partial z}+\frac{1}{2 r_{h}}+\alpha\left(\frac{1}{n_{o s}} \frac{d n_{o s}}{d z}+3 \gamma_{o s}^{2} u_{o s} \frac{d u_{o s}}{d z}\right)\right] \delta u_{s}=0 .
\end{aligned}
$$


In the similar way, we obtain from the conservation of entropy, (11),

$$
\delta P_{s}=\frac{\gamma_{g} P_{o s}}{n_{o s}} \delta n_{s}
$$

and from the total energy density, (34),

$$
\delta \rho_{s}=\frac{\rho_{o s}}{n_{o s}}\left(1+\frac{\gamma_{o s}^{2} \gamma_{g} P_{o s}}{\rho_{o s}}\right) \delta n_{s}+2 u_{o s} \gamma_{o s}^{2} \rho_{o s} \delta u_{s}
$$

where $\rho_{o s}=\gamma_{o s}^{2}\left(m_{s} n_{o s}+\Gamma_{g} P_{o s}\right)$. Linearizing the transverse part of the momentum conservation equation, differentiating it with respect to $t$ and then substituting from (30), we obtain

$$
\begin{gathered}
\left(\alpha u_{o s} \frac{\partial}{\partial z}+\frac{\partial}{\partial t}-\frac{u_{o s}}{2 r_{h}}+\frac{\mathrm{i} \alpha q_{s} \gamma_{o s} n_{o s} B_{o}}{\rho_{o s}}\right) \frac{\partial \delta v_{s}}{\partial t} \\
-\frac{\alpha q_{s} \gamma_{o s} n_{o s}}{\rho_{o s}}\left(\alpha u_{o s} \frac{\partial}{\partial z}+\frac{\partial}{\partial t}-\frac{u_{o s}}{2 r_{h}}\right) \delta E=0 .
\end{gathered}
$$

When linearized, Poisson's equation (29) and (32) respectively give

$$
\begin{gathered}
\frac{\partial \delta E_{z}}{\partial z}=4 \pi e\left(n_{o 2} \gamma_{o 2}-n_{o 1} \gamma_{o 1}\right)+4 \pi e\left(\gamma_{o 2} \delta n_{2}-\gamma_{o 1} \delta n_{1}\right) \\
+4 \pi e\left(n_{o 2} u_{o 2} \gamma_{o 2}^{3} \delta u_{2}-n_{o 1} u_{o 1} \gamma_{o 1}^{3} \delta u_{1}\right), \\
\left(\alpha^{2} \frac{\partial^{2}}{\partial z^{2}}+\frac{3 \alpha}{2 r_{h}} \frac{\partial}{\partial z}-\frac{\partial^{2}}{\partial t^{2}}+\frac{1}{4 r_{h}^{2}}\right) \delta E=4 \pi e \alpha\left(n_{o 2} \gamma_{o 2} \frac{\partial \delta v_{2}}{\partial t}-n_{o 1} \gamma_{o 1} \frac{\partial \delta v_{1}}{\partial t}\right) .
\end{gathered}
$$

\section{Dispersion Relation}

Our consideration effects on a local scale for which the distance from the horizon does not vary significantly. We use a local (or mean-field) approximation for the lapse function and hence for the equilibrium fields and fluid quantities. If the plasma is situated relatively close to the horizon, $\alpha^{2} \ll 1$, then a relatively small change in distance $z$ will make a significant difference to the magnitude of $\alpha$. Thus it is important to choose a sufficiently small range in $z$ so that $\alpha$ does not vary much.

We consider thin layers in the $\mathbf{e}_{\hat{z}}$ direction, each layer with its own $\alpha_{o}$, where $\alpha_{o}$ is some mean value of $\alpha$ within a particular layer. Then a more complete picture can be built up by considering a large number of layers within a chosen range of $\alpha_{o}$ values.

The local approximation imposes the restriction that the wavelength must be smaller in magnitude than the scale of the gradient of the lapse function $\alpha$, i.e., $\lambda<(\partial \alpha / \partial z)^{-1}=2 \ell$, or equivalently, $k>(\pi / \ell)$.

One of the disadvantages of the hydrodynamical approach is that it is essentially a bulk, fluid approach and therefore the microscopic behavior of the two-fluid plasma is treated in a somewhat approximate manner via the equation of state. It means that the results are really only strictly valid in the long wavelength limit. However, the restriction, imposed by the local approximation, on the wavelength is not too severe and permits the consideration of intermediate to long wavelengths so that the small $k$ limit is still valid.

In the local approximation for $\alpha, \alpha \simeq \alpha_{o}$ is valid within a particular layer. Hence, the unperturbed fields and fluid quantities and their derivatives, which are functions of $\alpha$, take on their corresponding "mean-field" values for a given $\alpha_{o}$. Then the coefficients in (44), (47) and (48) are constants within each 
layer with respect to $\alpha$ (and therefore $z$ as well). Hence, it is possible to Fourier transform the equations with respect to $z$, assuming plane-wave-type solutions for the perturbations of the form $\sim e^{(k z-\omega t)}$ for each $\alpha_{o}$ layer.

When Fourier transformed, (47) and (49) turn out to be

$$
\begin{gathered}
\delta E=\frac{\mathrm{i} 4 \pi e \alpha_{o} \omega\left(n_{o 2} \gamma_{o 2} \delta v_{2}-n_{o 1} \gamma_{o 1} \delta v_{1}\right)}{\alpha_{o} k\left(\alpha_{o} k-\mathrm{i} 3 / 2 r_{h}\right)-\omega^{2}-1 /\left(2 r_{h}\right)^{2}}, \\
\omega\left(\alpha_{o} k u_{o s}-\omega+\frac{\mathrm{i} u_{o s}}{2 r_{h}}+\frac{\alpha_{o} q_{s} \gamma_{o s} n_{o s} B_{o}}{\rho_{o s}}\right) \delta v_{s}-\mathrm{i} \alpha_{o} \frac{q_{s} \gamma_{o s} n_{o s}}{\rho_{o s}}\left(\alpha_{o} k u_{o s}-\omega-\frac{\mathrm{i} u_{o s}}{2 r_{h}}\right) \delta E=0 .
\end{gathered}
$$

The dispersion relation for the transverse electromagnetic wave modes may be written as

$$
\left[K_{ \pm}\left(K_{ \pm} \pm \frac{\mathrm{i}}{2 r_{h}}\right)-\omega^{2}+\frac{1}{\left(2 r_{h}\right)^{2}}\right]=\alpha_{o}^{2}\left\{\frac{\omega_{p 1}^{2}\left(\omega-u_{o 1} K_{ \pm}\right)}{\left(u_{o 1} K_{\mp}-\omega-\alpha_{o} \omega_{c 1}\right)}+\frac{\omega_{p 2}^{2}\left(\omega-u_{o 2} K_{ \pm}\right)}{\left(u_{o 2} K_{\mp}-\omega+\alpha_{o} \omega_{c 2}\right)}\right\}
$$

for either the electron-positron or electron-ion plasma. Here, $K_{ \pm}=\alpha_{o} k \pm \mathrm{i} / 2 r_{h}, \omega_{c s}=e \gamma_{o s} n_{o s} B_{o} / \rho_{o s}$, and $\omega_{p s}=\sqrt{4 \pi e^{2} \gamma_{o s}^{2} n_{o s}^{2} / \rho_{o s}}$. The cyclotron frequency $\omega_{c s}$, as well as the plasma frequency $\omega_{p s}$, is frame independent. Although the fluid quantities are measured in the fluid frame, the field $B_{o}$ is measured in the FIDO frame. Hence, the factors of $\gamma_{o s}$ do not cancel out explicitly. The transformation $B_{o} \rightarrow \gamma_{o s} B_{o}$ boosts the fluid frame for either fluid and thereby cancels the $\gamma_{o s}$ factors. The + and - denote the left $L$ and right $R$ modes, respectively. The complex conjugate of the dispersion relation for the $R$ mode gives the dispersion relation for the $L$ mode. In the special relativistic case, the two modes have the same dispersion relation.

\section{Numerical Solution Modes}

The dispersion relations (52) are complicated enough even in the simplest cases for the electron-positron plasma where both species are assumed to have the same equilibrium parameters, and an analytical solution is cumbersome and unprofitable. We therefore solve numerically the dispersion relation in order to determine all the physically meaningful modes for the transverse waves. We put the equations in the form of a matrix equation as follows:

$$
(A-k I) X=0,
$$

where the eigenvalue is chosen to be the wave number $k$, the eigenvector $X$ is given by the relevant set of perturbations, and $I$ is the identity matrix.

In order to write the perturbation equations in an appropriate form, we introduce the following set of dimensionless variables:

$$
\begin{aligned}
& \tilde{\omega}=\frac{\omega}{\alpha_{o} \omega_{*}}, \quad \tilde{k}=\frac{k c}{\omega_{*}}, \quad k_{h}=\frac{1}{2 r_{h} \omega_{*}}, \\
& \delta \tilde{u}_{s}=\frac{\delta u_{s}}{u_{o s}}, \quad \tilde{v}_{s}=\frac{\delta v_{s}}{u_{o s}}, \quad \delta \tilde{n}_{s}=\frac{\delta n_{s}}{n_{o s}}, \\
& \delta \tilde{B}=\frac{\delta B}{B_{o}}, \quad \tilde{E}=\frac{\delta E}{B_{o}}, \quad \delta \tilde{E}_{z}=\frac{\delta E_{z}}{B_{o}} .
\end{aligned}
$$

For an electron-positron plasma, $\omega_{p 1}=\omega_{p 2}$ and $\omega_{c 1}=\omega_{c 2}$; so, $\omega_{*}$ is defined as

$$
\omega_{*}=\left\{\begin{array}{l}
\omega_{c}, \quad \text { Alfvén modes, } \\
\left(2 \omega_{p}^{2}+\omega_{c}^{2}\right)^{\frac{1}{2}}, \text { high frequency modes, }
\end{array}\right.
$$


where $\omega_{p}=\sqrt{\omega_{p 1} \omega_{p 2}}$ and $\omega_{c}=\sqrt{\omega_{c 1} \omega_{c 2}}$. However, for the case of an electron-ion plasma, the plasma frequency and the cyclotron frequency are different for each fluid; so, the choice of $\omega_{*}$ is a more complicated matter. We assume, for simplicity, that

$$
\omega_{*}= \begin{cases}\frac{1}{\sqrt{2}}\left(\omega_{c 1}^{2}+\omega_{c 2}^{2}\right)^{\frac{1}{2}}, & \text { Alfvén modes, } \\ \left(\omega_{* 1}^{2}+\omega_{* 2}^{2}\right)^{\frac{1}{2}}, & \text { high frequency modes, }\end{cases}
$$

where $\omega_{* s}^{2}=\left(2 \omega_{p s}^{2}+\omega_{c s}^{2}\right)$.

The dimensionless eigenvector for the transverse set of equations is

$$
\tilde{X}_{\text {transverse }}=\left[\begin{array}{l}
\delta \tilde{v}_{1} \\
\delta \tilde{v}_{2} \\
\delta \tilde{B} \\
\delta \tilde{E}
\end{array}\right]
$$

When linearized and Fourier transformed, equations (30) and (31) turn out to be

$$
\begin{gathered}
\left(k-\frac{\mathrm{i}}{2 r_{h} \alpha_{o}}\right) \delta E+\frac{\mathrm{i} \omega}{\alpha_{o}} \delta B=0, \\
\frac{\mathrm{i} \omega}{\alpha_{o}} \delta E=\left(k-\frac{\mathrm{i}}{2 r_{h} \alpha_{o}}\right) \delta B+4 \pi e\left(\gamma_{o 2} n_{o 2} \delta v_{2}-\gamma_{o 1} n_{o 1} \delta v_{1}\right) .
\end{gathered}
$$

Using (54), we write (51), (58), and (59) in the dimensionless form:

$$
\begin{aligned}
\tilde{k} \delta \tilde{v}_{s} & =\left(\frac{\tilde{\omega}}{u_{o s}}-\left(\frac{q_{s}}{e}\right) \frac{\omega_{c s}}{u_{o s} \omega_{*}}-\frac{\mathrm{i} k_{h}}{\alpha_{o}}\right) \delta \tilde{v}_{s}+\left(\frac{q_{s}}{e}\right) \frac{\omega_{c s}}{u_{o s} \omega_{*}} \delta \tilde{B}-\mathrm{i}\left(\frac{q_{s}}{e}\right) \frac{\omega_{c s}}{u_{o s} \omega_{*}} \delta \tilde{E}, \\
\tilde{k} \delta \tilde{E} & =-\mathrm{i} \tilde{\omega} \delta \tilde{B}+\frac{\mathrm{i} k_{h}}{\alpha_{o}} \delta \tilde{E} \\
\tilde{k} \delta \tilde{B} & =u_{o 1} \frac{\omega_{p 1}^{2}}{\omega_{c 1} \omega_{*}} \delta \tilde{v}_{1}-u_{o 2} \frac{\omega_{p 2}^{2}}{\omega_{c 2} \omega_{*}} \delta \tilde{v}_{2}+\frac{\mathrm{i} k_{h}}{\alpha_{o}} \delta \tilde{B}+\mathrm{i} \tilde{\omega} \delta \tilde{E} .
\end{aligned}
$$

These are the equations in the required form to be used as input to (53).

\section{Results}

We carried out the numerical analysis using the well known MATLAB. We have considered both the electron-positron plasma and the electron-ion plasma. The limiting horizon values for the electronpositron plasma are taken to be

$$
n_{h s}=10^{18} \mathrm{~cm}^{-3}, \quad T_{h s}=10^{10} \mathrm{~K}, \quad B_{h}=3 \times 10^{6} \mathrm{G}, \quad \text { and } \quad \gamma_{g}=\frac{4}{3} .
$$

For the electron-ion plasma, the ions are essentially non relativistic, and the limiting horizon values are chosen to be

$$
n_{h 1}=10^{18} \mathrm{~cm}^{-3}, \quad T_{h 1}=10^{10} \mathrm{~K} ; \quad n_{h 2}=10^{15} \mathrm{~cm}^{-3}, \quad T_{h 2}=10^{12} \mathrm{~K} .
$$

The equilibrium magnetic field has the same value as it has for the electron-positron case. The gas constant is $\gamma_{g}=4 / 3$. 


\subsection{Alfvén Modes}

\subsubsection{Electron-Positron Plasma}

For the ultrarelativistic electron-positron plasma in the special relativistic case, only one purely real Alfvén mode exists [4], while for the Schwarzschild case there are two Alfvén modes [33]. In our analysis we find three Alfvén modes, as shown in Fig. 1 and Fig. 2., for the electron-positron plasma. The Alfvén modes in de Sitter space are interesting in that, there exists three Alfvén modes for the electron-positron plasma compared with four modes for the electron-ion plasma. These three modes for the electronpositron plasma coalesce into a single mode on taking the special relativistic limit, giving the result of ref. 41. Since we have used the convention $e^{\mathrm{i} k z}=e^{\mathrm{i}[\operatorname{Re}(k)+\mathrm{i} \operatorname{Im}(k)]}$, the damping corresponds to $\operatorname{Im}(\tilde{k})>0$ and growth to $\operatorname{Im}(\tilde{k})<0$.

\subsubsection{Electron-Ion Plasma}

In this case four modes are found, two of which are growth and the other two are damped. The modes shown in Fig. 3 and Fig. 4 are damped and the remaining two modes shown in Fig. 5 and Fig. 6 are growth. The first two modes are equivalent to the modes shown in Fig. 1 and the other two are equivalent to the modes shown in Fig. 2 for electron-positron plasma.

The differences in the magnitudes of the $\omega_{c 1}$ and $\omega_{c 2}$ for the first two modes apparently lead to take the frequencies from their negative (and therefore unphysical) values for the electron-positron case to positive physical values for the electron-ion case. These changes are thus because of the difference in mass and density factors as between the positrons and ions.

These four modes for electron-ion plasma are equivalent to those of the Schwarzschild case [33]. It is evident that the growth and damping rates are independent of the frequency, but depended only on the value of $\alpha_{o}$.

\subsection{High Frequency Modes}

\subsubsection{Electron-Positron Plasma}

In this case three high frequency electromagnetic modes are found for the electron-positron plasma, as shown in Figs. 7-9. High frequency modes in the horizon of dS space for each fluid are interesting in that all the modes are both damping and growth modes.

The two modes, shown in Figs. 7 and 8, are similar and both modes are damping and growth very near to the horizon. These two modes are growth for most of the frequency domain but shows damped for lower frequencies as $\omega \rightarrow .04$ and $\alpha_{o} \rightarrow 0$. Thus at a distance from the horizon corresponding to $\alpha_{o} \rightarrow 0.2$ it appears that energy is no longer fed into wave mode by the gravitational field but begins to be drained from the waves. The third mode shown in Fig. 9 is also growth and damping mode. This mode is damped for most of the frequency domain but shows growth for lower frequencies as $\omega \rightarrow .04$ and $\alpha_{o} \rightarrow 0$. These three modes are equivalent to the three modes of ref. [33] for this case. Also these three modes coalesce with a single modes in the special relativistic case [41] as $\alpha_{o} \rightarrow 1$. 


\subsubsection{Electron-Ion Plasma}

Similar as for the electron-positron plasma, the electron-ion plasma has three high frequency modes. Two of these, shown in Fig. 10 and Fig. 11, shows damping and growth very near to the horizon for lower frequencies, and for upper frequencies they are growth modes. These two modes are growth for higher frequency but shows damped for lower frequencies as $\omega \rightarrow .02$ and $\alpha_{o} \rightarrow 0$. The third mode, shown in Fig. 12, is growth for lower frequencies as $\omega \rightarrow .02$ and $\alpha_{o} \rightarrow 0$ and shows damped for higher frequencies. These three modes are similar to the modes for high frequency electron-ion plasma in the Schwarzschild case [33].

\section{Concluding Remarks}

The main concern of this study has been exclusively the investigation, within the local approximation, of Alfvén and high frequency transverse electromagnetic waves in a two-plasma in the purely de Sitter space. We derive the dispersion relations for the Alfven and high frequency electromagnetic waves by using a local approximation and give their numerical solutions. In the limit $\ell \rightarrow 0$ our results reduce to that in special relativity as obtained by Sakai and Kawata [41] (i.e., only one purely real mode for Alfvén and high frequency electromagnetic waves). In contrast to the work of Sakai and Kawata 41, new modes (damped or growth) arise for the Alfven and high frequency electromagnetic waves in the pure dS space. In our work all the modes for Alfvén waves are either damped or growing, but for high frequency electromagnetic waves all the modes are both damped and growing. This is because of the singularity of the de Sitter space. For the electron-positron plasma, the damping and growth rates are similar with the electron-ion plasma but different by several orders of magnitude, compared with the real components of the wave number. For both the fluid components the damping and growth rates are obviously frequency independent, but are dependent on the radial distance from the horizon as denoted by the mean value of the lapse function $\alpha_{o}$. This is of course not for the case of the high frequency waves. In that case the rate of damping or growth is dependent on both frequency and radial distance from the horizon. Damped modes demonstrate, at least in this approximation, that energy is being drained from some of the waves by the gravitational field. The majority of the modes are growth rates and that indicate that the gravitational field is feeding energy into the waves.

In the light of recent astronomical observations, it has been suggested that our universe will asymptotically approach a de Sitter space [1. Hence, aspects of the de Sitter space might be of interest in a broader context. Our study of plasmas in the de Sitter space is thus well motivated.

\section{Acknowledgement}

One of the authors (MHA) thanks the SIDA as well as the Abdus Salam International Centre for Theoretical Physics (ICTP), Trieste, Italy, for supporting with an Associate position of the Centre. 


\section{References}

[1] N. Bahcall, J. P. Ostriker, S. Perlmutter, and P. J. Steinhardt, Science 284, (1999)1481.

[2] A.G. Reiss, et al., Astron. J. 116, (1998)1009.

[3] S. Perlmutter, et al., Astron. J. 517, (1999)565.

[4] J.P. Ostriker, P.J. Steinhardt, Nature 377, (1995)600.

[5] S. Perlmutter et al., Astrophys. J. 483, (1997)565.

[6] B. Schmidt et al., Astrophys. J. 507, (1998)46.

[7] J. Maldacena, Adv. Theor. Math. Phys. 2, (1998)231.

[8] E. Witten, Adv. Theor. Math. Phys. 2, (1998)253.

[9] S. Gubser, I. Klebanov, and A. Polyakov, Phys. Lett. B 428, (1998)105.

[10] O. Aharony, S. Gubser, J. Maldacena, H. Ooguri, and Y. Oz, Phys. Rep. 323, (2000)183.

[11] E. Witten, "Quantum gravity in de Sitter space,"hep-th/0106109.

[12] A. Strominger, JHEP 0110, (2001)034.

[13] A. Strominger, JHEP 0111, (2001)049.

[14] D. Klemm, Nucl. Phys. B, 625, (2002)295.

[15] C. M. Hull, J. High Energy Phys. 07, (1998)021.

[16] Mu-In Park, Phys. Lett. B 440, (1998)275.

[17] Mu-In Park, Nucl. Phys. B 544, (1999)377.

[18] I. Antoniadis, P. Mazur, and E. Mottola, astro-ph/9705200

[19] A. Volovich, hep-th/0101176.

[20] V. Balasubramanian, P. Horava, and D. Minic, J. High Energy Phys. 05, (2001)043.

[21] C.M. Hull, and R.R. Khuri, Nucl. Phys. B 575, (2000)231.

[22] P.O. Mazur and E. Mottola, Phys. Rev. D 64, (2001)104022.

[23] M. Spradlin, A. Strominger, and A. Volovich, "Les Houches Lectures on De Sitter Space,"hep-th/0110007

[24] B. McInnes, Nucl. Phys. B 627, (2002)311.

[25] R. Bousso, A. Maloney, and A. Strominger, Phys. Rev. D 65, (2002)104039. 
[26] M. Spradlin and A. Volovich, Phys. Rev. D 65, (2002)104037.

[27] A. J. M. Medved, Phys. Rev. D 66, (2002)124009.

[28] M. Parikh, Phys. Lett. B 546, (2002)189.

[29] R. Bousso, A. Maloney, and A. Strominger, Phys. Rev. D 65, (2002)104039.

[30] Kyung-Seok Cha, Bum-Hoon Lee, and Chanyong Park, J. Korean Phys. Soc. 42, (2003)735 hep-th/0207194.

[31] S. Nojiri and S. Odintsov, J. High Energy Phys. 12, (2001)033.

[32] S. Nojiri and S. Odintsov, Phys. Lett. B 523, (2001)165.

[33] V. Buzzi, K. C. Hines, R. A. Treumann: Phys. Rev. D 51, (1995)6663.

[34] V. Buzzi, K. C. Hines, R. A. Treumann: Phys. Rev. D 51, (1995)6677.

[35] K.S. Thorne, D.A. Macdonald: Mon. Not. R. Astron. Soc. 198,(1982)339.

[36] D.A. Macdonald, K.S. Thorne: Mon. Not. R. Astron. Soc. 198,(1982)345.

[37] R.H. Price, K.S. Thorne: Phys. Rev. D 33,(1986)915.

[38] K.S. Thorne, R.H. Price, D.A. Macdonald: Black Holes: The Membrane Paradigm. Yale University Press, New Haven (1986).

[39] E. Harris: Phys. Rev. 108,(1957)1357.

[40] F. Jüttner: Ann. Phys. 34,(1911)856(Leipzig).

[41] J. Sakai, T. Kawata: J. Phys. Soc. Jpn. 49,(1980)747. 

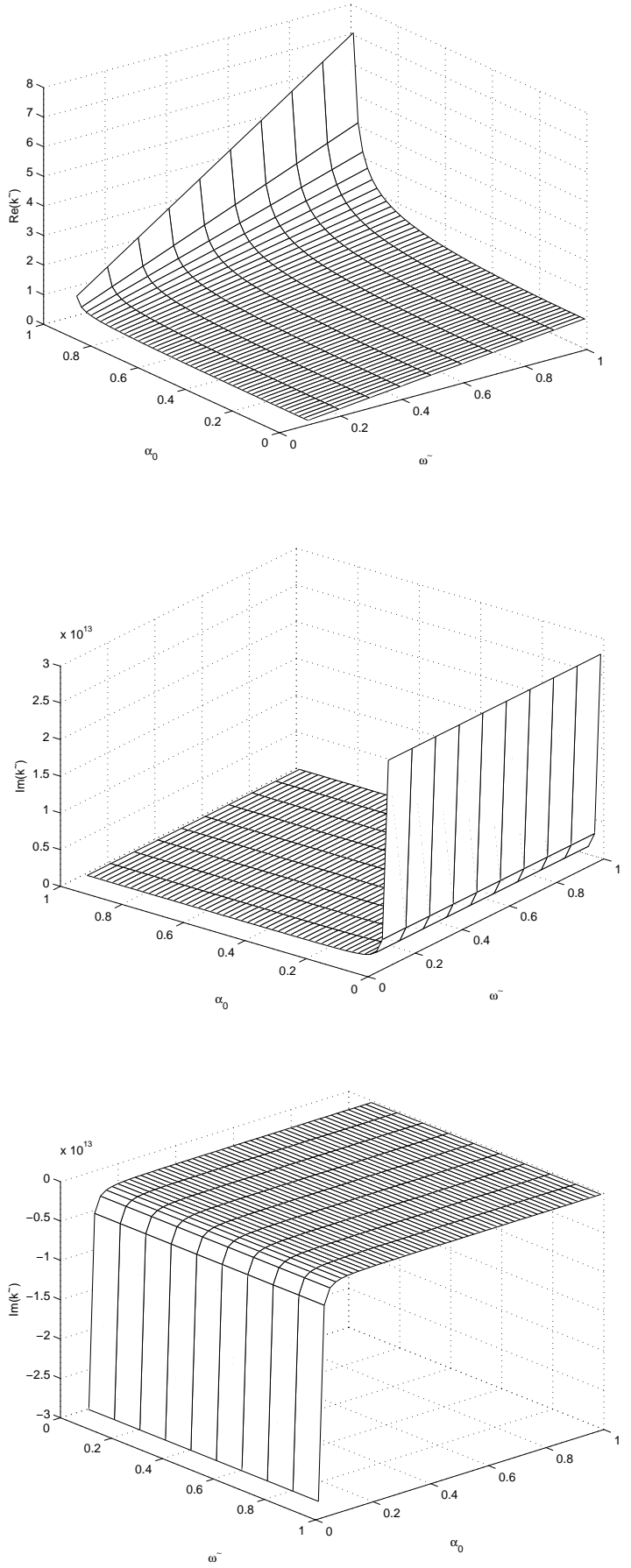

Figure 1: Top: Real part of Alfvén mode for the electron-positron plasma. Middle: Imaginary part of Alfvén damped mode. Bottom: Imaginary part of Alfvén growth mode. 



Figure 2: Left: Real part of Alfvén mode for the electron-positron plasma. Right: Imaginary part of Alfvén growth mode.
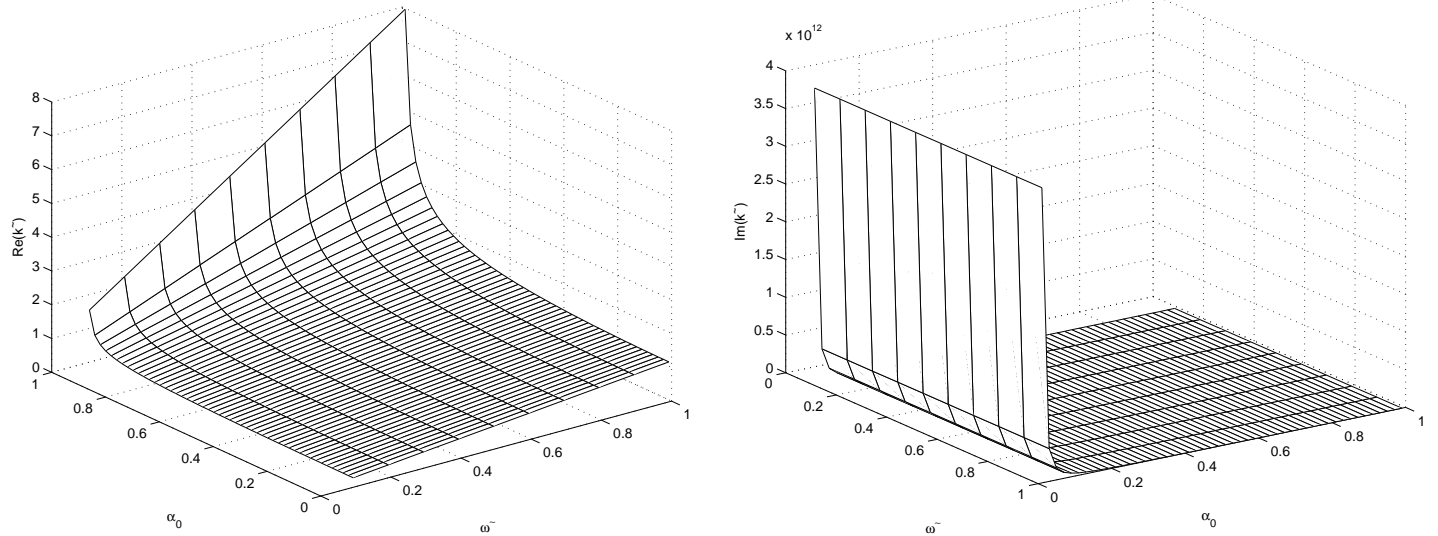

Figure 3: Left: Real part of Alfvén mode for the electron-ion plasma. Right: Imaginary part of Alfvén damped mode.
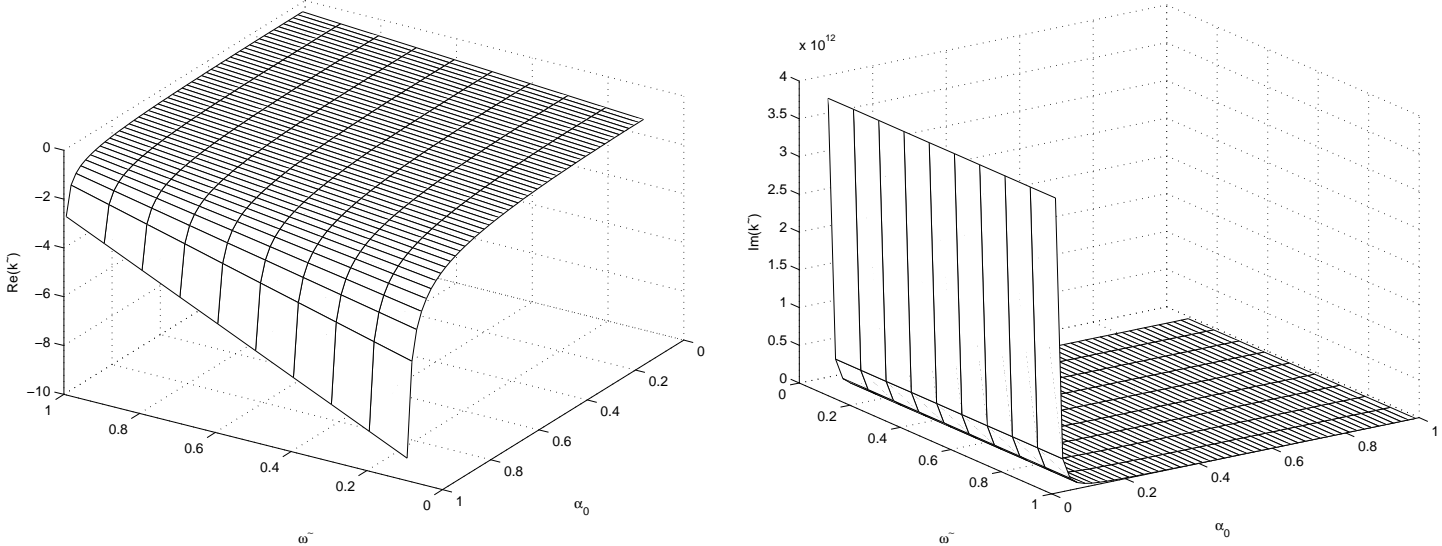

Figure 4: Left: Real part of Alfvén mode for the electron-ion plasma. Right: Imaginary part of Alfvén damped mode. 

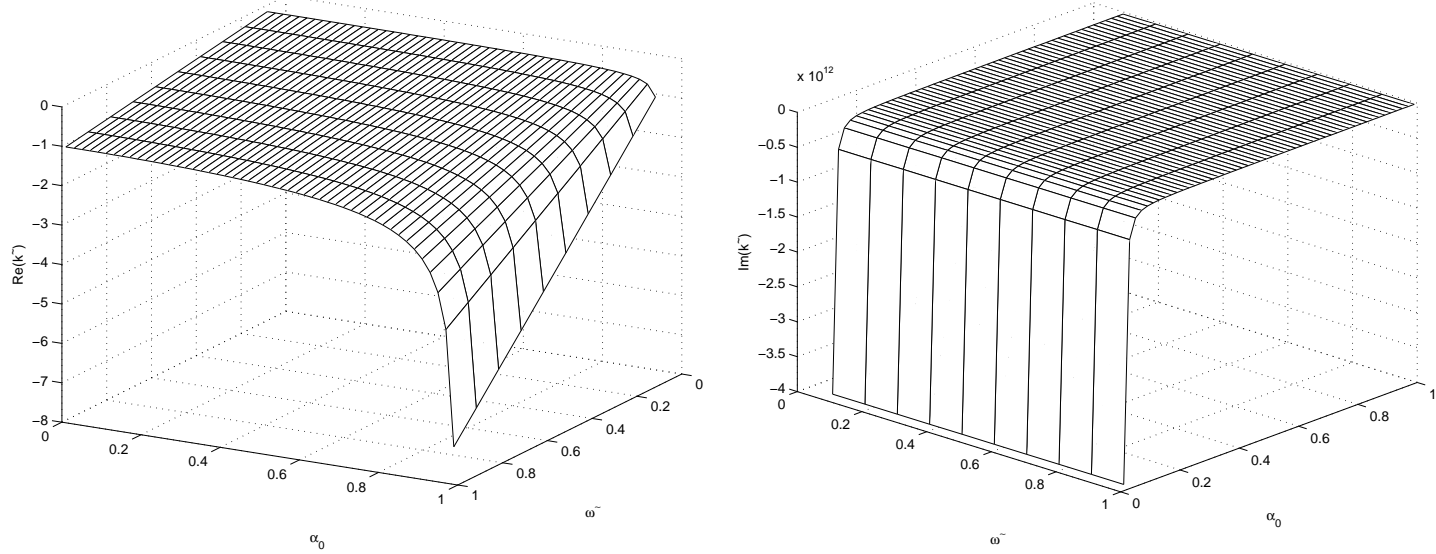

Figure 5: Left: Real part of Alfvén mode for the electron-ion plasma. Right: Imaginary part of Alfvén growth mode.
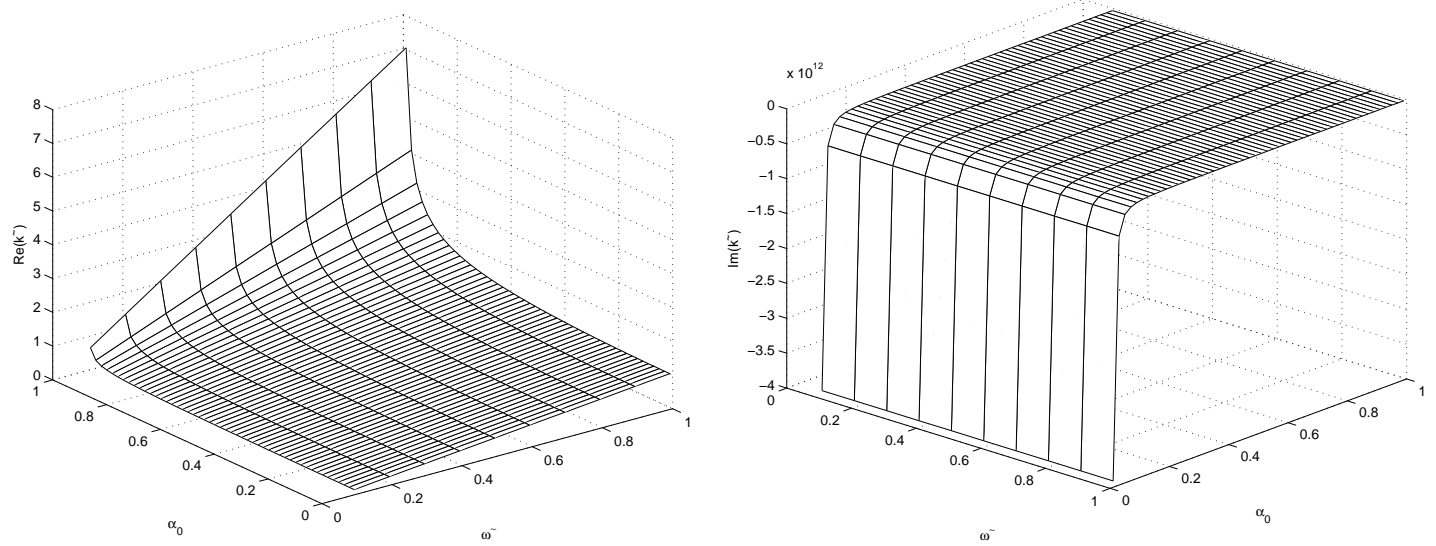

Figure 6: Left: Real part of Alfvén mode for the electron-ion plasma. Right: Imaginary part of Alfvén growth mode.
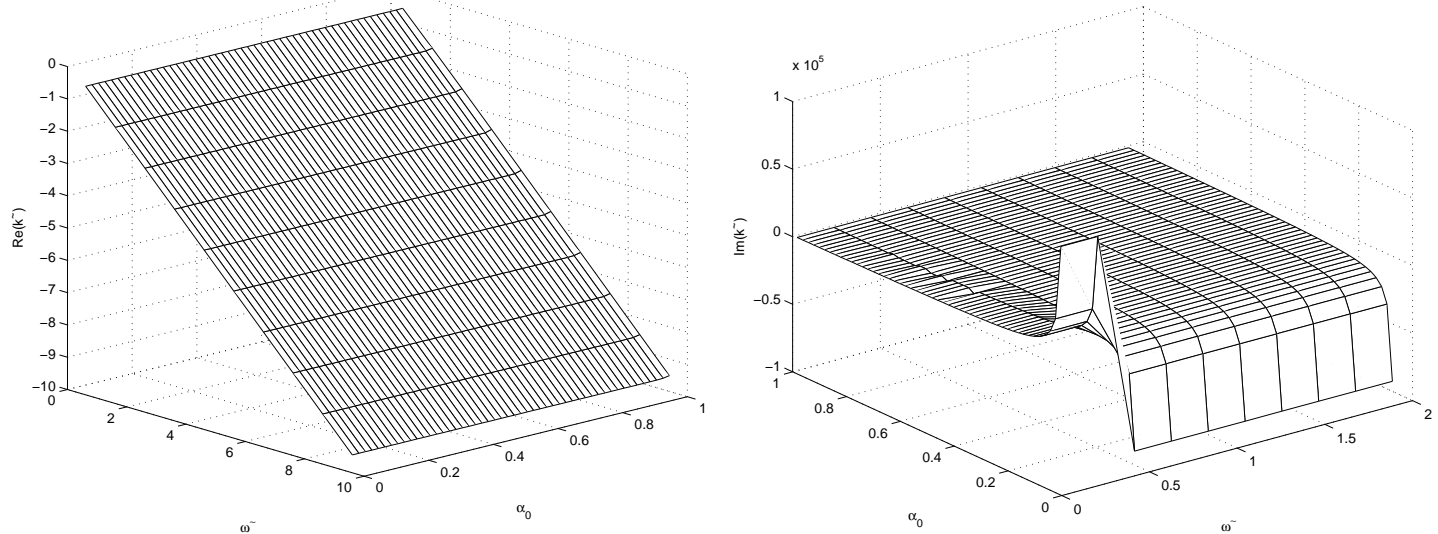

Figure 7: Left: Real part of high frequency mode for the electron-positron plasma. Right: Imaginary part of high frequency damping and growth mode. 

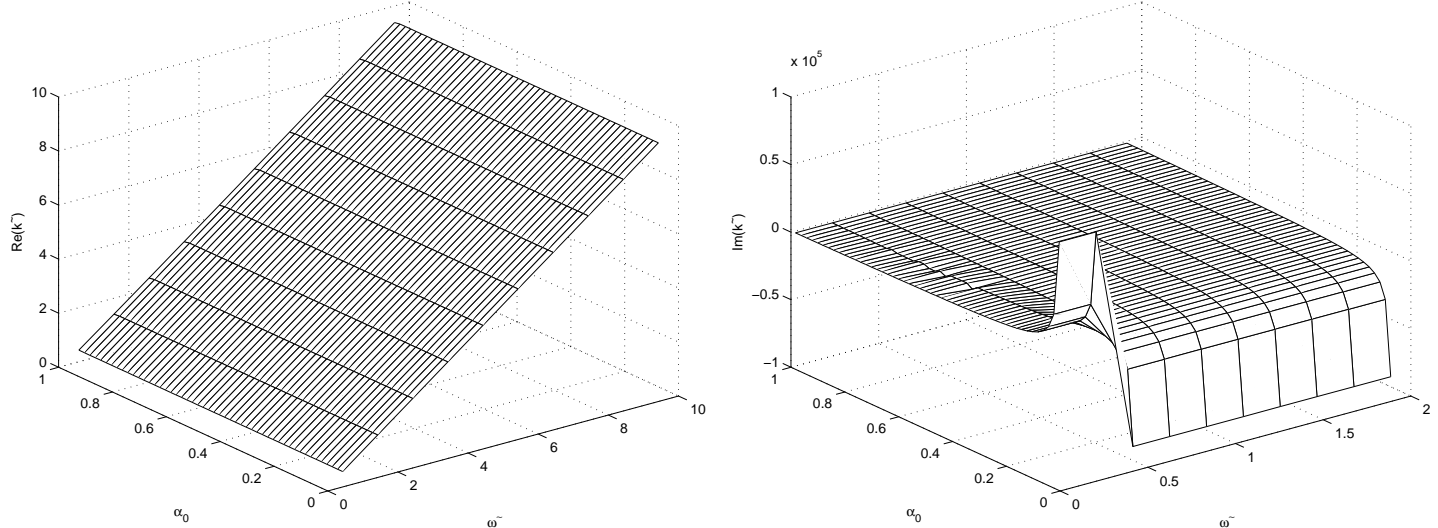

Figure 8: Left: Real part of high frequency mode for the electron-positron plasma. Right: Imaginary part of high frequency damping and growth mode.
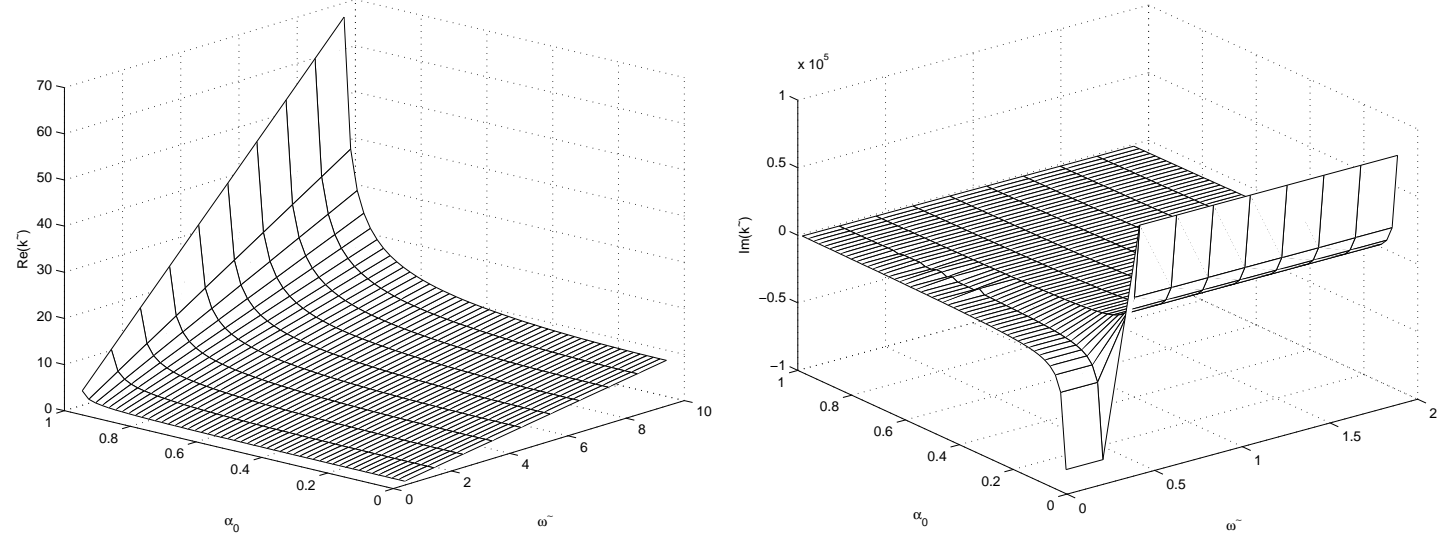

Figure 9: Left: Real part of high frequency mode for the electron-positron plasma. Right: Imaginary part of high frequency growth and damping mode.
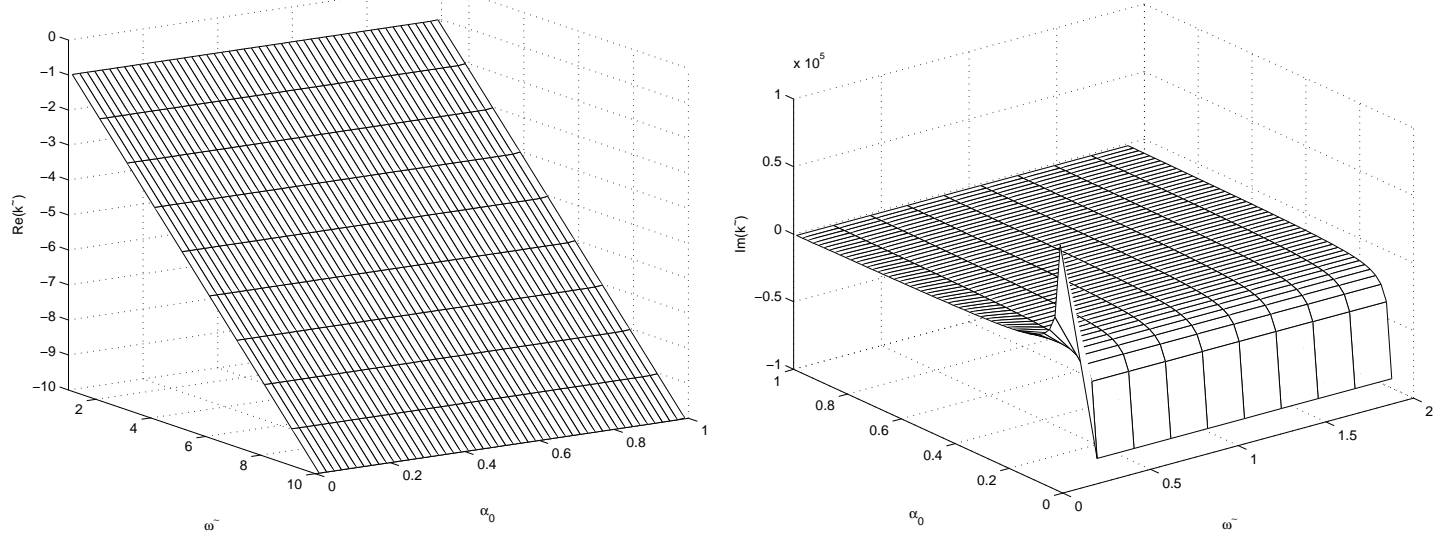

Figure 10: Left: Real part of high frequency mode for the electron-ion plasma. Right: Imaginary part of high frequency damping and growth mode. 

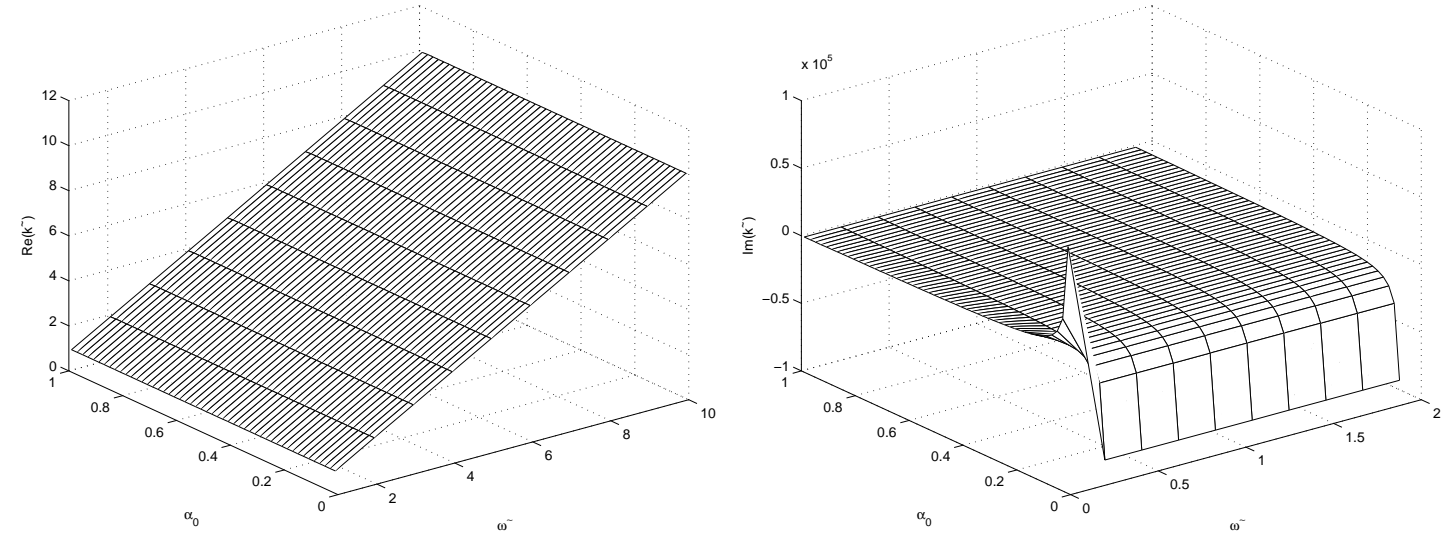

Figure 11: Left: Real part of high frequency mode for the electron-ion plasma. Right: Imaginary part of high frequency damping and growth mode.
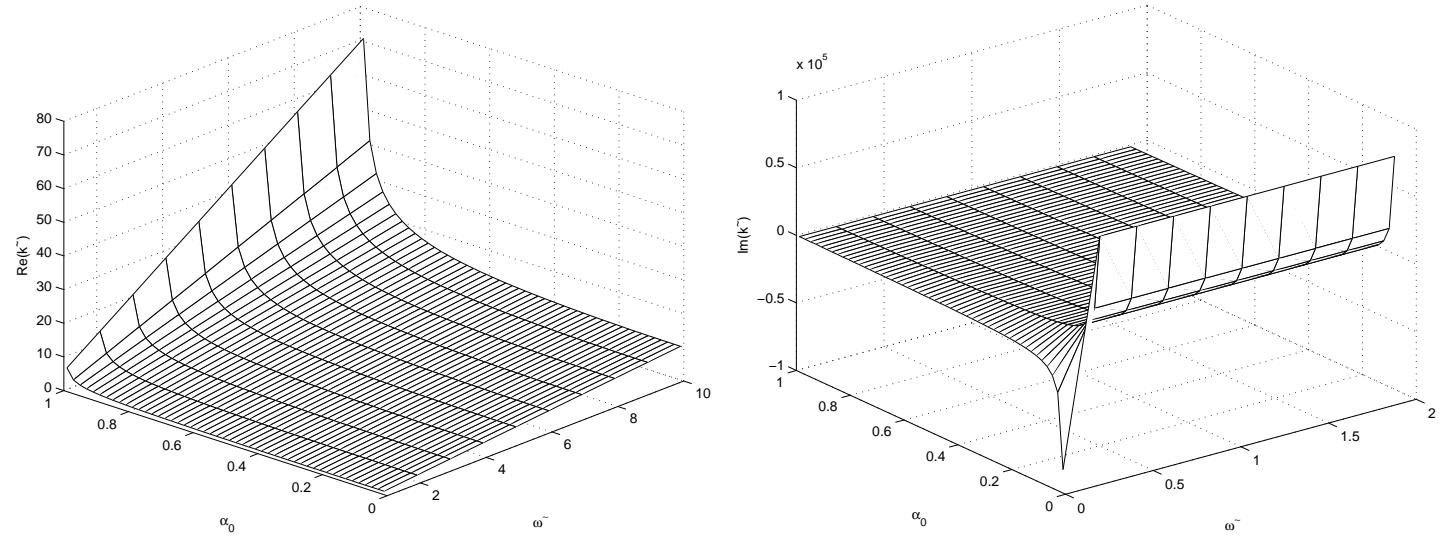

Figure 12: Left: Real part of high frequency mode for the electron-ion plasma. Right: Imaginary part of high frequency growth and damping mode. 